\title{
Mass Transfer Flow Through an Inclined Plate with Porous Medium
}

\author{
Manjiul Islam, Farjana Akter, Ariful Islam \\ Mathematics Discipline, Khulna University, Khulna, Bangladesh
}

Email address:

manjiul.math@gmail.com (M. Islam),munni091211@gmail.com (F. Akter), is_arif@yahoo.com (A. Islam)

To cite this article:

Manjiul Islam, Farjana Akter, Ariful Islam. Mass Transfer Flow Through an Inclined Plate with Porous Medium. American Journal of Applied Mathematics. Vol. 3, No. 5, 2015, pp. 215-220. doi: 10.11648/j.ajam.20150305.12

\begin{abstract}
The numerical studies are performed to examine the mass transfer flow through porous medium with an inclined plate. The governing partial differential equations are transformed to a system of dimensionless coupled partial differential equation. Finite difference technique is used as a tool for the numerical approach. The corresponding momentum, concentration and continuity equation are derived by employing the usual transformation, and finite difference method has been used to solve the above equations. The effects on the velocity and concentration distribution of various parameters entering into the problem separately are discussed with the help of graphs and tables.
\end{abstract}

Keywords: Mass Transfer, Inclined Plate, Schmidt Number, Porous Medium

\section{Introduction}

Investigation of mass transfer flow has attracted the interest of many researchers in view of its important applications of Mass Transfer include the dispersion of contaminants, drying and humidifying, segregation and doping in materials, vaporization and condensation in a mixture, evaporation (boiling of a pure substance is not mass transfer), combustion and most other chemical processes, cooling towers, sorption at an interface (adsorption) or in a bulk (absorption), and most living-matter processes as respiration (in the lungs and at cell level), nutrition, secretion, sweating, etc. In many engineering application, combine heat and mass transformation play a vital role in fluid condensing or boiling at a solid surface. The combine heat and mass transformation consideration arise due to buoyancy forces caused by mass diffusions. The study of Sparrow et al. (1959) is related to the convection flow about an inclined surface in which the combined forced and free boundary layer problem has been discussed [1]. Boundary layer flow due to continuously moving surface as propounded by Sakiadis (1961) and its further varied extensions proved to be significant designing and study tools [2]. Gebhart and Pera (1971) studied mass transfer effects on free convection flow past a semi-infinite plate [3]. Soundalgekar and Ganesan (1981) was analyzed natural free convection with mass transfer on an Isothermal flat plate [4]. The vertical free convection boundary layer flow in porous medium owing to combined heat and mass transfer has been investigated by Bejan and Khair (1985) [5]. Lai and Kulacki (1990) used the series expansion method to investigate coupled heat and mass transfer in natural convection from a sphere in a porous medium [6]. The next year Lai and Kulacki (1991) studied Coupled heat and mass transfer by natural convection from vertical surfaces in porous media [7]. Elbashbeshy (1997) studied mass transfer along a vertical plate in the presence of magnetic field [8].

The study of the effect of mass transfer on Newtonian and non-Newtonian fluids has become important in the last few years. This importance is due to a number of industrial processes. For instance the food processing, biochemical operations and transport polymers. Flowing over deformable boundaries has also gained importance because of its immediate practical application in lubrication technology, biophysical flows and many other transportation types. Besides, the characteristics of the flow of blood through arteries and veins are of considerable medical interest. Chamkha et al. (2000) studied the effects of Hydro magnetic combined heat and mass transfer by natural convection from a permeable surface embedded in a fluid saturated porous medium [9]. Chamkha and Khaled (2001) investigated the problem of coupled heat and mass transfer from an inclined plate in the presence of absorption [10]. An analytical solution for unsteady free convection in porous media has been studied by Magyari et al. (2004) [11]. Elgazery, (2008) numerically analyzed the heat and mass transfer by natural convection in 
power law fluid past a vertical plate immersed in a porous medium [12]. Recently Bhuvaneswari et al. (2010) studied exact analysis of radiation convective flow heat and mass transfer over an inclined plate in a porous medium [13]. Heat and mass transfer in porous medium is very much prevalent in nature and can also be encountered in many manmade technological processes, that is why theory of flow through porous media has emerged as a vibrant discipline of intensive research activity.

Hence our aim to study Mass transfer flow through an inclined plate with porous medium. The dimensionless equations are solved numerically. The effects of various physical parameters such as Schmidt number $S_{c}$, Modified Grashof number $G_{m}$ and Permeability parameter $k$ on the velocity and concentration are shown graphically. The results allow us to predict the different behavior for the velocity and concentration distributions that can be observed when the relevant parameters are varied.

\section{Mathematical Model of Flow}

By introducing Cartesian co-ordinate system, the $\mathrm{X}$-axis is chosen along the plane in the direction of the flow and the Y-axis is normal to it. Initially it has been considered that the plate as well as the fluid is at the same concentration level $C\left(C_{\infty}\right)$ everywhere in the fluid is same. Also it is considered that the fluid and the plate is at rest after that the plate is to be moving with a constant velocity. $U_{0}$ in its own. Plane and instantaneously at time $t>0$, the species concentration of the plate are raised to $C_{w}\left(>C_{\infty}\right)$, which are there after maintained constant, where $C_{w}$ are species concentration at the wall of the plate and $C_{\infty}$ are the concentration of the species far away from the plate.

Within the framework of the above stated assumptions with reference to the generalized equations described before the equation relevant to the transient two dimensional problems are governed by the following system of coupled non-linear differential equations.

Continuity Equation

$$
\frac{\partial u}{\partial x}+\frac{\partial v}{\partial y}=0
$$

\section{Momentum Equation}

$$
\frac{\partial \mathrm{u}}{\partial \mathrm{t}}+u \frac{\partial \mathrm{u}}{\partial \mathrm{x}}+v \frac{\partial \mathrm{u}}{\partial \mathrm{y}}=g \beta\left(c-c_{\infty}\right) \cos \alpha+v\left(\frac{\partial^{2} \mathrm{u}}{\partial \mathrm{y}^{2}}\right)-\frac{v}{k^{\prime}} u
$$

\section{Concentration Equation}

$$
\frac{\partial c}{\partial t}+u \frac{\partial c}{\partial x}+v \frac{\partial c}{\partial y}=D_{c} \frac{\partial^{2} c}{\partial y^{2}}
$$

With the corresponding initial and boundary conditions are At $t=0 u=0, v=0, c \rightarrow c_{\infty}$ everywhere

$$
\begin{array}{r}
u=0, v=0, c \rightarrow c_{\infty} \text { at } x=0 \\
t>0 u=U_{0}, v=0, c \rightarrow c_{w} \text { at } y=0 \\
u=0, v=0, c \rightarrow c_{\infty} \text { at } y \rightarrow \infty
\end{array}
$$

Where $x, y$ are Cartesian co-ordinate $u, v$ and $x, y$ component of flow velocity respectively. Here $g$ is the local acceleration due to gravity; $\vartheta$ is the kinetic viscosity; $\rho$ is the density of the fluid, $D$ is the coefficient of mass diffusivity.

\section{Mathematical Formulation}

Since the solution of the governing equations under the initial and boundary conditions will be based on a finite difference method it is required to make the said equations dimensionless.

For this purpose it has been now introduced the following dimensionless variables;

$$
\begin{aligned}
& X=\frac{x U_{0}}{v}, Y=\frac{y U_{0}}{v}, U=\frac{u}{U_{0}}, V=\frac{v}{U_{0}}, \\
& \tau=\frac{t U_{0}^{2}}{v}, \text { and } C=\frac{c-c_{\infty}}{c_{w}-c_{\infty}}
\end{aligned}
$$

Where $\tau$ represents the dimensionless time, $X \& Y$ be the dimensionless Cartesian coordinates, Where $U$ and $V$ be the dimensionless velocity components and $C$ be the dimensionless concentration.

Using the above relation, we obtain the following non-dimensional coupled partial differential equation,

$$
\begin{gathered}
\frac{\partial U}{\partial X}+\frac{\partial V}{\partial Y}=0 \\
\frac{\partial U}{\partial \tau}+U \frac{\partial U}{\partial X}+V \frac{\partial U}{\partial Y}=\frac{\partial^{2} U}{\partial Y^{2}}+G_{m} C \cos \alpha-k U \\
\frac{\partial C}{\partial \tau}+U \frac{\partial C}{\partial X}+V \frac{\partial C}{\partial Y}=\frac{1}{S_{C}} \frac{\partial^{2} C}{\partial Y^{2}}
\end{gathered}
$$

Where,

$G_{m}=v g \beta \frac{\left(C_{w}-C_{\infty}\right)}{U_{0}^{3}}$ (Modified Grashof Number)

$k=\frac{U^{2}}{k^{\prime} U_{0}^{2}}$ (Permeability Parameter)

$S_{C}=\frac{v}{D}$ (Schimdth Number)

Also the associate initial and boundary condition become At $\tau=0 U=0, V=0, C=0$ everywhere

$$
\begin{array}{r}
U=0, V=0, C=0 \text { at } X=0 \\
\tau>0 \quad U=1, V=0, C=1 \text { at } Y=0 \\
U=0, V=0, C=0 \text { at } Y \rightarrow \infty
\end{array}
$$




\section{Numerical Solution}

The explicit finite difference method has been used to solve the governed second order nonlinear coupled dimensionless partial differential equations with the corresponding initial and boundary conditions. To obtain a system of finite difference equations, the flow region is divided into a grid or meshes of lines parallel to $X$ and $Y$ axes where $X$-axis is taken along the plate and $Y$-axis is normal to the plate. Here it is considered that $X_{\max }(=400)$ i.e. $X$ varies from 0 to 400 and regard $Y_{\max }(=25)$ as corresponding to $Y \rightarrow \infty$ i.e. $Y$ varies from 0 to 25 . It is also considered that $m=60$ and $n=60$ grid spacing in the $X$ and $Y$ directions respectively. We

have the constant mesh size along $X$ direction, $\Delta X=6.67(0 \leq X \leq 400)$ and the constant mesh size along $Y$ direction, $\Delta Y=0.42(0 \leq Y \leq 25)$ with the smaller time-step $\Delta \tau=0.05$.

Let $U^{\prime}, V^{\prime}$ and $C^{\prime}$ denote the values of $U, V$ and $C$ at the end of a time-step respectively. Using the finite difference approximations we obtain the following set of finite difference equations,

$$
\frac{U_{i, j}-U_{i-1, j}}{\Delta X}+\frac{V_{i, j}-V_{i, j-1}}{\Delta Y}=0
$$

$$
\begin{gathered}
\frac{U_{i, j}^{\prime}-U_{i, j}}{\Delta \tau}+U_{i, j} \frac{U_{i, j}-U_{i-1, j}}{\Delta X}+V_{i, j} \frac{U_{i, j+1}-U_{i, j}}{\Delta Y}=G_{m} C_{i, j} \cos \alpha+\frac{U_{i, j+1}-2 U_{i, j}+U_{i, j-1}}{(\Delta Y)^{2}}-K U_{i, j} \\
\frac{C_{i, j}^{\prime}-C_{i, j}}{\Delta \tau}+U_{i, j} \frac{C_{i, j}-C_{i-1, j}}{\Delta X}+V_{i, j} \frac{C_{i, j+1}-C_{i, j}}{\Delta Y}=\frac{1}{S_{C}} \frac{C_{i, j+1}-2 C_{i, j}+C_{i, j-1}}{(\Delta Y)^{2}}
\end{gathered}
$$

And the initial and boundary conditions with finite difference scheme are;

$$
\begin{array}{r}
U_{i, j}^{0}=0, \quad V_{i, j}^{0}=0, \quad C_{i, j}^{0}=0, \\
U_{0, j}^{n}=0, \quad V_{0, j}^{n}=0, \quad C_{0, j}^{n}=0, \\
U_{i, 0}^{n}=1, \quad V_{i, 0}^{n}=0, \quad C_{i, 0}^{n}=1, \\
U_{i, L}^{n}=0, \quad V_{i, L}^{n}=0, \quad C_{i, L}^{n}=0,
\end{array}
$$

Where, $L \rightarrow \infty$

Here the subscripts $i$ and $j$ designate the grid points $x$ and $y$ coordinates respectively and the superscript $n$ represents a value of time, $\tau=n \Delta \tau$ where, $n=0,1,2,3, \ldots$ From the initial condition, the values of $U$ is known at $\tau=0$. Then at the end of the any time-step $\Delta \tau$, the new concentration $C^{\prime}$, the new velocity $U^{\prime}$ and $V^{\prime}$ at all interior nodal points may be obtained by successive application of concentration and momentum equation respectively. This process is repeated in time and provided the time-step is sufficiently small, hence $U, V$ and $C$ should eventually convergent values which approximate the steady-state solution of the problem.

\section{Results and Discussions}

To discuss the effects of the associated non-dimensional parameters on the flow variables, the numerical solutions of the problem are obtained by the explicit finite difference method with the help of a computer programming language Compaq Visual Fortran. In order to analyze the physical solution of the model, we have computed the steady numerical values of the non-dimensional Velocity $U$ and Concentration $C$ within the boundary layer for different values of Modified Grashofnumber $\left(G_{m}\right)$, Inclination $(\alpha)$, Schimdth number
$\left(S_{c}\right)$ and Permeability parameter $(k)$. Hence the velocity and concentration profile are drawn for $\tau=30,60$ and 80 .

The effect of the Modified Grashof number on the velocity field is presented in Fig. 5.1. It is observed that the velocity increases with the rise of $G_{m}$. It is observed from Fig. 5.2 that the velocity decreases in case of increasing Inclination. The same effect on the velocity curve is found in Fig. 5.3 that is the velocity decreases with increase of Schimdth number. A decreasing effect of Permeability parameter found on Fig. 5.4.

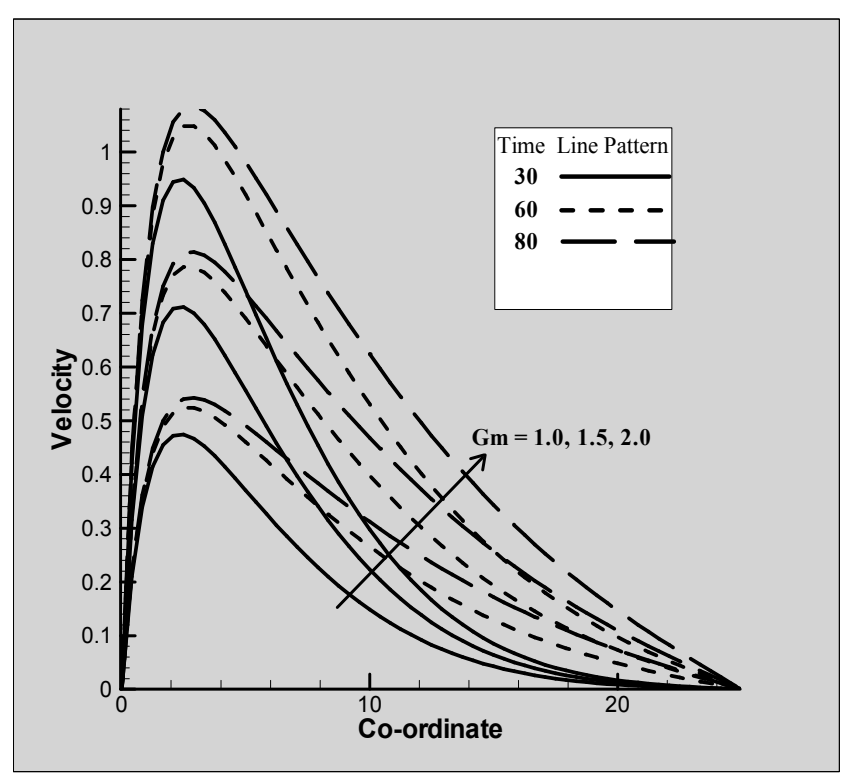

Fig. 5.1. Velocity profiles for $\alpha=45^{0}, S_{C}=0.94, k=0.3$. 


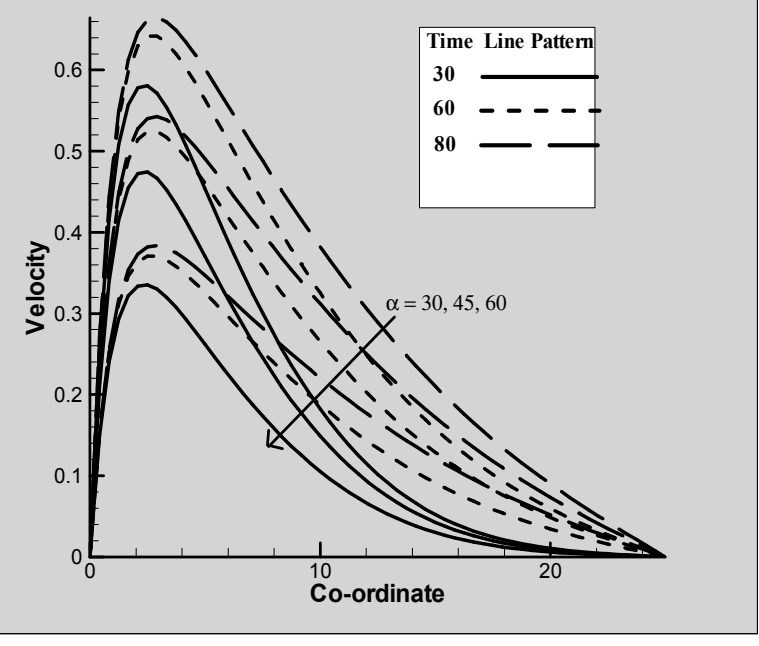

Fig. 5.2. Velocity profiles for $G_{m}=1.0, S_{C}=0.94, k=0.3$

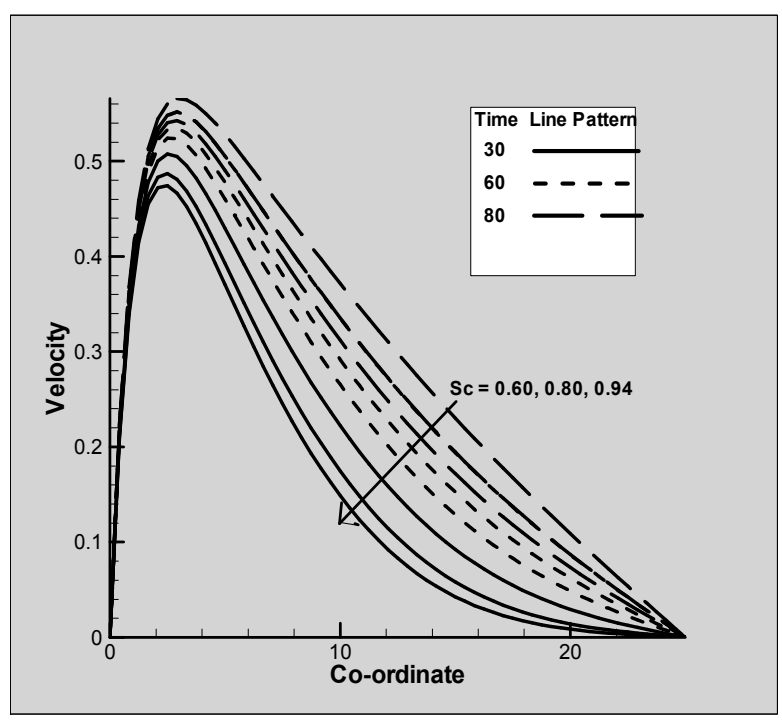

Fig. 5.3. Velocity profiles for $G_{m}=1.0, \quad \alpha=45^{0}, k=0.3$.

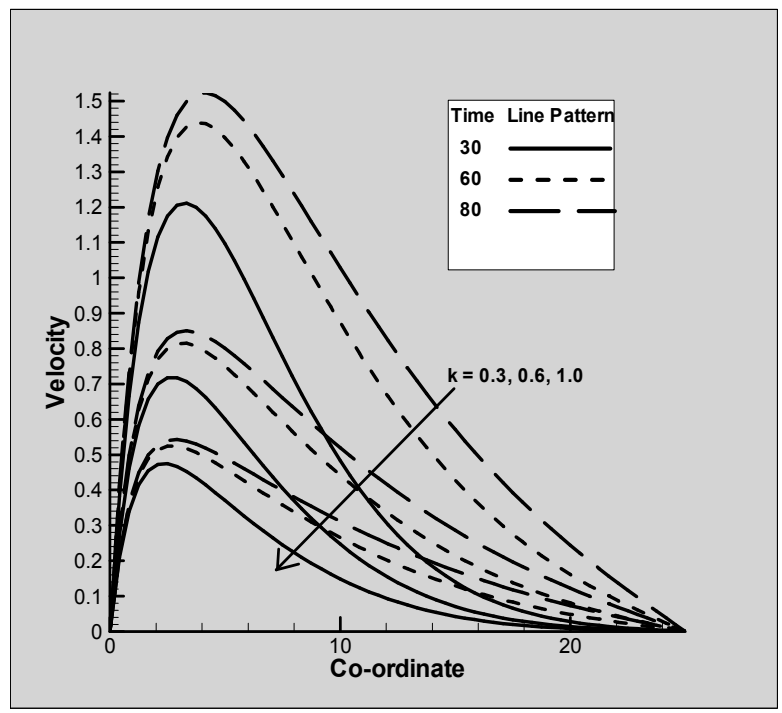

Fig. 5.4. Velocity profiles for $G_{m}=1.0, \alpha=45^{0}, S_{C}=0.94$.

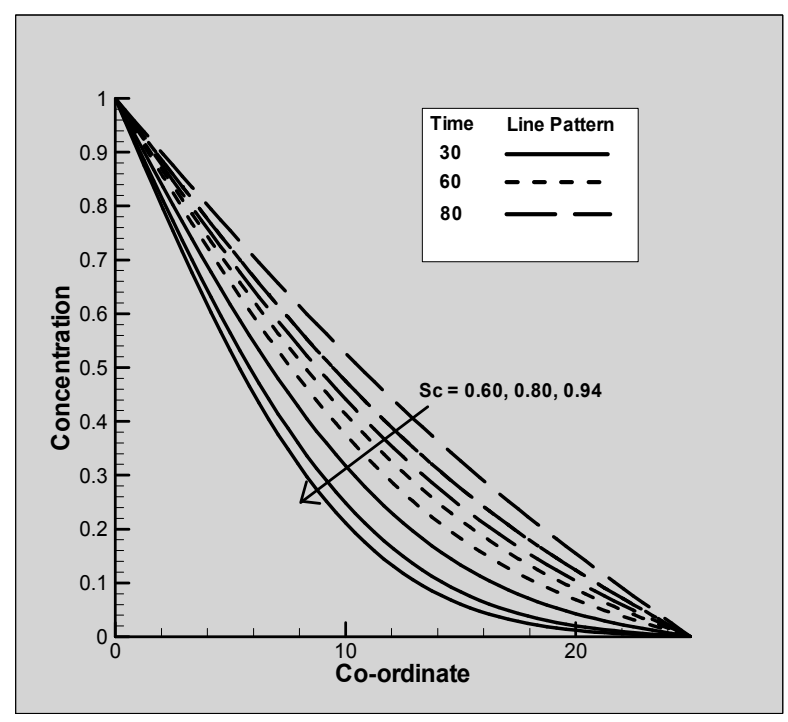

Fig. 5.5. Concentration profiles for $G_{m}=1.0, \quad \alpha=45^{0}, \quad k=0.3$

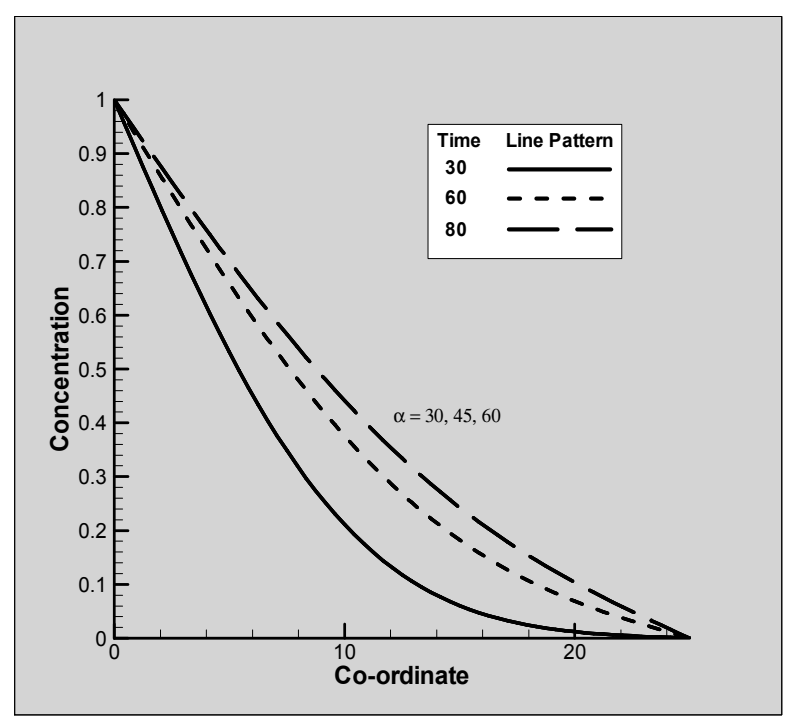

Fig. 5.6. Concentration profiles for $G_{m}=1.0, S_{C}=0.94, k=0.3$.

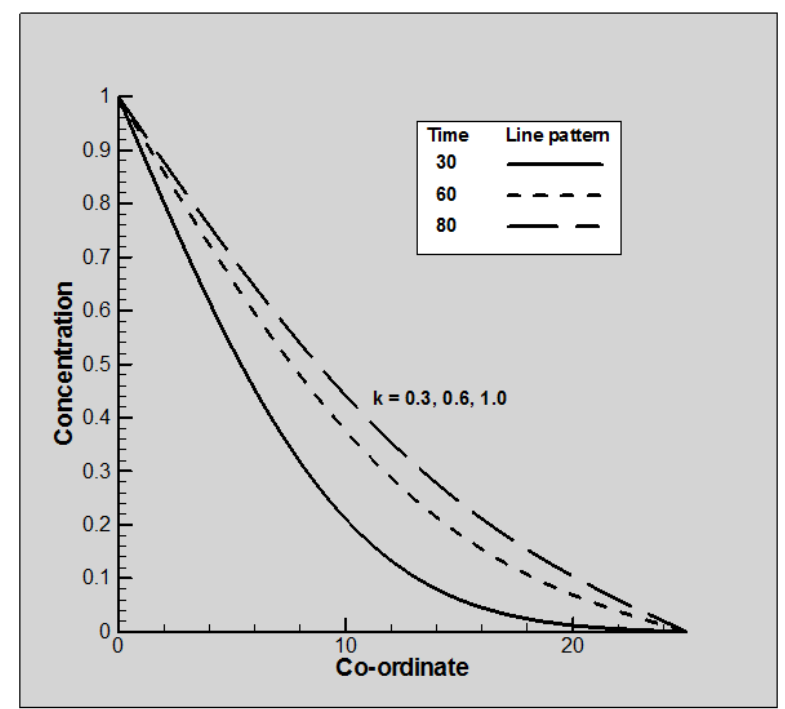

Fig. 5.7. Concentration profiles for $G_{m}=1.0, \quad \alpha=45^{0}, \quad S_{C}=0.94$ 


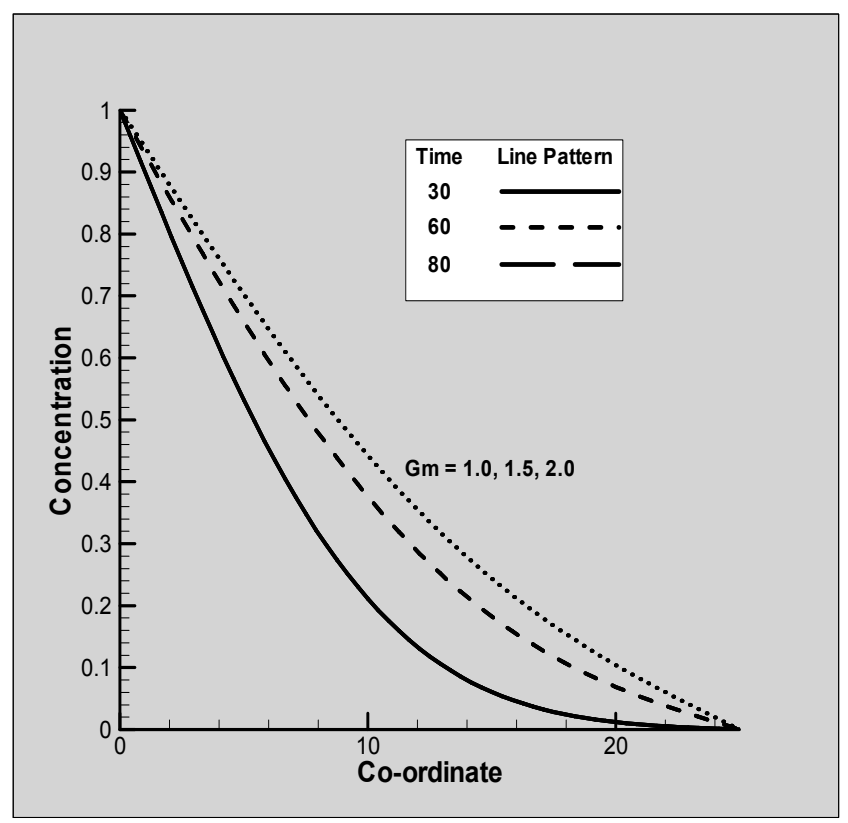

Fig. 5.8. Concentration profiles for $\alpha=45^{0}, S_{C}=0.94, k=0.3$.

Fig.5.5 shows that the steady state concentration of fluid decreases with the increase of Schimdt number. In Fig.5.6.we see that the fluid concentration remain unchanged with the increasing value of Inclination. An unchanged effect of Permeability parameter on concentration curves are observed in Fig.5.7. The effect of Modified Grashof number on concentration profiles are shown in Fig. 5.8.

\section{Conclusions}

Some of the important findings obtained from the graphical representation of the results are listed below:

The velocity distribution decreases with the increase of Schmidt number $\left(S_{c}\right)$ also the concentration distribution decreases with the increase of Schmidt number $\left(S_{c}\right)$.

The velocity distribution decreases with the increase of inclined angle $(\alpha)$ while the concentration distribution remains unchanged with the variety of inclined angle $(\alpha)$.

The Concentration distribution remains unchanged with the increase of Modified Grashof number $\left(G_{m}\right)$ while the velocity distribution increases with the increase of Modified Grashof number $\left(G_{m}\right)$.

The velocity distribution decreases with the increase of Permeability parameter $(k)$ while the concentration distribution remains unchanged with the variety of Permeability parameter $(k)$.

\section{Acknowledgement}

The research project has supported Mathematics discipline, Khulna University. Therefore we have expressed gratefulness to Mathematics discipline, Khulna University for providing lab facilities.

\section{Nomenclature}

$\begin{array}{ll}S_{c} & \text { Schmidt number } \\ G_{m} & \text { Modified Grashof number } \\ k & \text { Permeability parameter } \\ C & \text { Concentration } \\ D_{c} & \text { Mass Diffusivity } \\ u & \text { Velocity component in the x-direction } \\ v & \text { Velocity component in the y-direction } \\ w & \text { Velocity component in the z-direction } \\ \nabla & \text { Differential operator } \\ \alpha & \text { Angle of inclination } \\ \beta & \text { Co-efficient of thermal expansion } \\ \delta & \text { Boundary layer thickness } \\ \mu & \text { Co-efficient of viscosity } \\ \vartheta & \text { Co-efficient of kinematic viscosity } \\ \rho & \text { Density of the fluid }\end{array}$

\section{References}

[1] E. M. Sparrow, R. Eichhorn and J. L. Grigg, (1959), Combined forced and free convection in a boundary layer, Physics of Fluids, 2:319-320.

[2] B. C. Sakiadis, (1961), Boundary layer behavior on continuous solid surfaces, AI Ch E J, 7, 26-28.

[3] B. Gebhart and L. Pera, (1971), The nature of vertical natural convection flows resulting from the combined buoyancy effect of thermal and mass diffusion, International Journal of Heat and Mass Transfer, 14:2025-2050.

[4] Soundalgekar and Ganesan P (1981), Finite-differential analysis of transient free convection with mass transfer on an isothermal vertical plate, International journal of engineering and science, 19:757-770.

[5] Bejan, A. and Khair, K. R., (1985), Heat and Mass transfer by natural convection in a porous medium, International Journal of Heat and Mass Transfer, 28:909-918.

[6] Lai, F. C. and Kulachi, F. A., (1990), The effects of variable viscosity on convective heat transfer along a vertical surface in a saturated porous medium, International Journal of Heat and Mass Transfer, 33:1028-1031.

[7] Lai, F. C. and Kulacki, F. A., (1991), Coupled heat and mass transfer by natural convection from vertical surfaces in porous media, International Journal of Heat Mass Transfer, 24:1189-1194.

[8] Elbashbeshy, (1997), Mass transfer along a vertical plate, International Journal of Heat and Mass Transfer, 19:165.

[9] Chamaka A. J., (2000), International Journal of Num. Methods for heat and fluid flow, 10(5): 455-476.

[10] Chamka and Khaled, (2001), Simultaneously heat and mass transfer in free convection, Industrial Engineering Chemical, 49:961-968.

[11] Magyari E. Pop I and Keller B., (2004), Analytical solutions for unsteady free convection flow through a porous media, Journal of Engineering mathematics, 48:93-104. 
[12] Naseer S. Elgazery, (2008), Transient analysis of heat and mass transfer by natural convection in power law fluid past a vertical plate immersed in a porous medium (numerical study), Applications and Applied Mathematics, 3(2):267-285.
[13] Bhuvaneswari, M. S. Sivasankaran and Y. J. Kim, (2010), Exact analysis of radiation convective flow heat and mass transfer over an inclined plate in a porous medium, World Applied Journal, 10:774-778. 